\title{
SPECTRAL DECOMPOSITIONS OF ONE-PARAMETER GROUPS OF ISOMETRIES ON HARDY SPACES
}

\author{
DIMITRI KARAYANNAKIS
}

\begin{abstract}
Spectral decompositions of strongly continuous one-parameter groups of surjective isometries on Hardy spaces of the disk $\mathbf{D}$ and the torus $\mathbf{T}^{2}$ are examined; a concrete description of the (pointwise) action of these decompositions is presented, mainly in the parabolic case, leading to a complete description of the action of the partial sum-operators of $\mathrm{M}$. Riesz when carried from $L^{p}(\mathbf{R})$ to $H^{p}(\mathbf{D}), 1<p \leq 2$. The (pointwise) action of the spectral decompositions of these isometric groups on $H^{p}\left(\mathbf{T}^{2}\right), 1<p<+\infty$, is also examined and concrete descriptions are derived, mainly in the parabolic case.
\end{abstract}

\section{INTRODUCTION}

It is well known that in the general $L^{p}$ setting, $1<p<+\infty, p \neq 2$ projection valued measures cannot give rise to spectral decompositions needed in order to describe a large number of harmonic analysis phenomena. (For definitions, see $\S 2$.)

In [1], H. Benzinger, E. Berkson, and T. A. Gillespie, adopting an abstract operator-theoretic approach that avoids vector measures, achieved a generalization to arbitrary Banach spaces of classical Stone's theorem for one-parameter unitary groups.

Departing even further from the typical setting of projection valued measures E. Berkson, T. A. Gillespie and P. S. Muhly [5] recently developed an abstract spectral theory for Banach spaces possessing the unconditionality property for martingale differences, i.e. for UMD spaces (see e.g. [9]).

The following result [5, Theorem (5.5)] will be instrumental in the core of this work:

Theorem. Let $\left\{T_{t}\right\}, t \in \mathbf{R}$, be a uniformly bounded strongly continuous oneparameter group of operators on a UMD space $X$. Then there is a unique spectral family $\varepsilon(\cdot)$ in $X$, called the Stone-type spectral family of $\left\{T_{t}\right\}, t \in \mathbf{R}$, such that

Received by the editors June 6, 1987.

1980 Mathematics Subject Classification (1985 Revision). Primary 30D55, 47D05; Secondary 42A $45,43 \mathrm{~A} 50$.

Key words and phrases. Isometry, group of isometries, Hardy space, spectral family, group of Möbius transformations, projection, residue theory.

This paper constitutes a revised portion of the author's Ph.D. dissertation written under Professor Earl Berkson at the University of Illinois. 
for any $t \in \mathbf{R}$

$$
T_{t}=\operatorname{st}_{a \rightarrow+\infty} \int_{-a}^{a} e^{i t \lambda} d \varepsilon(\lambda)
$$

(as a Stieltjes integral, and where "st" indicates the strong operator topology of $\beta(X))$.

In particular (1.1) is applicable to one-parameter groups of isometeries on reflexive $L^{p}$-subspaces such as $H^{p}(\mathbf{D})$ and $H^{p}\left(\mathbf{T}^{2}\right), 1<p<+\infty$, which will be the focus of this paper.

In $\S 3$ we will obtain a concrete method for calculating the pointwise action of $\varepsilon(\cdot)$ in (1.1), mainly for isometric groups induced by one-parameter parabolic groups of Möbius transformations of D, via F. Forelli's representation [13], on $X=H^{p}(\mathbf{D})$. To achieve this goal we will introduce a suitable norm-dense subspace $Y$ of $H^{p}(\mathbf{D})$ and we will employ contour integration techniques.

As applications of this concrete description of $\left.\varepsilon(\lambda)\right|_{Y}, \lambda \in \mathbf{R}$ (Theorem (3.5)), we will reproduce-independently, and by rather elementary means-the results in [2] concerning $\varepsilon(\lambda) H^{p}(\mathbf{D})$ (Corollary (3.7)); we will describe concretely the action of the partial sum-operators of $\mathrm{M}$. Riesz when carried from $L^{p}(\mathbf{R})$ to $H^{p}(\mathbf{D}), 1<p \leq 2$ (Theorem (3.8)), along with the action of "F. Riesz-B. Nagy operators" (introduced in [20] and used in [1]) for the case of parabolic isometric groups in $H^{p}(\mathbf{D}), 1<p \leq 2$ (Corollary (3.10)); in addition, the inner-outer factorization of $(\varepsilon(\lambda) f)(z)$ for $f(z) \in Y$, in the parabolic case and $1<p<+\infty$, will be examined (Corollary (3.6)) and other related features that explore the role the above-mentioned spectral projections play in the structure of $H^{p}(\mathbf{D})$. Finally, in $\S 4$, we will obtain the corresponding results, mainly for the parabolic isometric group, on $X=H^{p}\left(\mathbf{T}^{2}\right), 1<p<+\infty$, using the representation of Berkson and Porta [8] and by introducing a suitable, for our purposes, direct sum decomposition of $H^{p}\left(\mathbf{T}^{2}\right)$ (Lemma (4.5)).

\section{Preliminaries}

We consider the definition and standard theory of $H^{p}(\mathbf{D})$ as contained in [12, 15 and 18] to be well known, as well as the concepts of an isometry on a Banach space $X$ and of a strongly continuous one-parameter group of operators in $X$; by the term "operator in $X$ " we always refer to a bounded linear transformation with domain the space $X$ and range in $X$, and we denote the algebra of all operators in $X$ by $\beta(X)$.

For ease of reference we give a brief account of concepts and known results required throughout this paper.

Definition [21]. A spectral family in a Banach space $X$ is a uniformly bounded projection valued function $F: \mathbf{R} \rightarrow \beta(X)$, such that

(i) $F(\lambda) F(\mu)=F(\mu) F(\lambda)=F(\min \{\lambda, \mu\})$ for any $\lambda, \mu \in \mathbf{R}$;

(ii) $F(\cdot)$ is st-right continuous on $\mathbf{R}$; 
(iii) $F(\cdot)$ has a st-left limit at each point of $\mathbf{R}$;

(iv) st- $\lim _{\lambda+\infty} F(\lambda)=I$ and st- $\lim _{\lambda \rightarrow-\infty} F(\lambda)=0$.

Definition. Let $\left\{\varphi_{t}\right\}, t \in \mathbf{R}$, be a family of univalent analytic maps of $\mathbf{D}$ onto D such that $\varphi_{t}$ is not the identity map for some $t$. We will call $\left\{\varphi_{t}\right\}$ a (nonconstant) one-parameter group of Möbius transformatins of $\mathbf{D}$ if

(i) $\varphi_{t} \circ \varphi_{s}=\varphi_{t+s}, t, s \in \mathbf{R}$;

(ii) $\varphi_{0}$ is the identity map on $\mathbf{D}$;

(iii) $\varphi_{t}(z)$ is continuous in $(t, z)$ on $(-\infty,+\infty) \times \mathbf{D}$.

It was shown in [7] that the set of common fixed points in the extended plane of such an $\left\{\varphi_{t}\right\}$ must be

(i) either a doubleton set, consisting of a point in $\mathbf{D}$ and its symmetric image w.r.t. the unit circle $\mathbf{T}$ (elliptic case); or (ii) a singleton subset of $\mathbf{T}$ (parabolic case); or finally (iii) a doubleton subset of $\mathbf{T}$ (hyperbolic case). In this paper we will focus attention only on the isometric groups induced by the parabolic and hyperbolic $\left\{\varphi_{t}\right\}, t \in \mathbf{R}$, and we will call them, simply, parabolic and hyperbolic isometric groups respectively.

We have the following results concerning $\varphi_{t}(z)[6,13]$ :

Proposition (2.1). Let $\left\{\varphi_{t}\right\}, t \in \mathbf{R}$, be a one-parameter (nonconstant) group of Möbius transformations of $\mathbf{D}$. Then

(a) if $\left\{\varphi_{t}\right\}$ is parabolic there are unique constants $c \in \mathbf{R} \backslash\{0\}$ and $a \in \mathbf{T}$ such that for $t \in \mathbf{R}, z \in \mathbf{D}$ we have

$$
\varphi_{t}(z)=\frac{(1-i c t) z+i c t a}{-i c \bar{a} t z+i+i c t},
$$

(b) if $\left\{\varphi_{t}\right\}$ is hyperbolic there are unique constants $c>0$ and $\alpha, \beta \in \mathbf{T}, \alpha \neq$ $\beta$, such that for $t \in \mathbf{R}, z \in \mathbf{D}$ and $\sigma_{\alpha, \beta}=(z-\alpha) /(z-\beta)$

$$
\varphi_{t}(z)=\sigma_{\alpha, \beta}^{-1}\left(e^{c t} \sigma_{\alpha, \beta}(z)\right) \text {. }
$$

Proposition (2.2). Let $\left\{T_{t}\right\}, t \in \mathbf{R}$, be a strongly continuous one-parameter group of isometries of $H^{p}(\mathbf{D}), 1<p<+\infty, p \neq 2$, not continuous in the uniform operator topology. Then $T_{t}$ has the unique representation

$$
T_{t} f=e^{i \omega t}\left(\varphi_{t}^{\prime}\right)^{1 / p} f\left(\varphi_{t}\right) \quad \text { for } t \in \mathbf{R}, f \in H^{p}(\mathbf{D})
$$

where $\omega \in \mathbf{R}$ and $\left\{\varphi_{t}\right\}$ is a one-parameter group of Möbius transformations of D. Conversely for such $\omega$ and $\left\{\varphi_{t}\right\}$ the above equation defines a one-parameter isometric group of $H^{p}(\mathbf{D}), 1<p<+\infty$, that is continuous in the strong but not in the uniform operator topology.

Remarks. (1) In the case $p=2$ we examine only the one-parameter group of isometries on $H^{2}(\mathbf{D})$ whose action is described in Proposition (2.2).

(2) We can always select a suitable analytic branch $\left\{\varphi_{t}^{\prime}\right\}^{1 / p}$ such that the composition rule for $\left\{\varphi_{s+t}^{\prime}\right\}^{1 / p}$ is valid. We standardize our notation by taking

$$
\left\{\varphi_{t}^{\prime}(z)\right\}^{1 / p}=\exp \frac{2}{P}\left\{\log \left(1-\varphi_{t}(z)\right)-\log (1-z)\right\}
$$

(see [6] for details). 
Following now [2, or 16], we have the following lemma on the "normalization" of $\left\{\varphi_{t}\right\}$ and its corresponding $\left\{T_{t}\right\}$ as described in Proposition (2.2).

Lemma (2.3). (a) Let $\left\{\varphi_{t}\right\}, t \in \mathbf{R}$, be as in Proposition (2.1(a)) and $\left\{n_{t}\right\}, t \in \mathbf{R}$, the corresponding (parabolic) group obtained by the substitutions $a=1, c=\frac{1}{2}$ in $\varphi_{t}(z)$. Let also $\left\{V_{t}\right\}, t \in \mathbf{R}$, be the one-parameter isometric group on $H^{p}(\mathbf{D})$ induced by $\left\{n_{t}\right\}, t \in \mathbf{R}$, as described in Proposition (2.2). Then $U_{1} T_{t} U_{1}^{-1}=$ $e^{i \omega t} V_{2 c t}$, where $U_{1}$ is the isometry on $H^{p}(\mathbf{D})$ defined by $\left(U_{1} f\right)(z)=f(a z), z \in$ D.

(b) Let $\left\{\varphi_{t}\right\}, t \in \mathbf{R}$, be as in Proposition (2.1(b)) and $\left\{\psi_{t}\right\}, t \in \mathbf{R}$, the corresponding (hyperbolic) group of Möbius transformations obtained by substituting $\alpha=-1, \beta=1$ and $c=1$ in $\varphi_{t}(z)$. Let also $\left\{Y_{t}\right\}, t \in \mathbf{R}$, be the isometric group induced by $\left\{\psi_{t}\right\}, t \in \mathbf{R}$, as in Proposition (2.2). If $U_{0}$ is the isometry on $H^{p}(\mathbf{D})$ induced by the Möbius transformation of $\mathbf{D}, \theta$, with $\theta(\alpha)=-1$ and $\theta(\beta)=1$, then there are constants $\delta \in \mathbf{R}$ and $\rho>0$ such that $V_{0}^{-1} T_{t} V_{0}=e^{i \delta t} Y_{\rho t}$.

In view of Lemma (2.3) (combined with Corollary (3.18) in [2]) we conclude that the study of the parabolic/hyperbolic group of isometries $\left\{T_{t}\right\}, t \in \mathbf{R}$, induced by the parabolic/hyperbolic group $\left\{\varphi_{t}\right\}$, as well as the study of their spectral families, reduces to the study of the group $\left\{V_{t}\right\}, t \in \mathbf{R}$, and its spectral family $E_{p}(\lambda)$ (resp. $\left\{Y_{t}\right\}, t \in \mathbf{R}$, and $F_{p}(\lambda)$ ); the above notation will be standarized throughout the rest of the paper.

We conclude this section with some results, considered classical, which we state without their proofs:

Lemma (2.4) [18]. $(1-z)^{\alpha} \in H^{p}(\mathbf{D})$ iff $\operatorname{Re} \alpha>-1 / p$.

Lemma (2.5) [14]. If $F \in H^{p}(\mathbf{D}), F \neq 0$ and $1 / F \in H^{r}(\mathbf{D})$ for some $r>0$, then $F$ is outer.

Lemma (2.6) [14]. If $F \in H^{p}(\mathbf{D})$ is outer then the $H^{p}(\mathbf{D})$-closure of $\operatorname{sp}\left\{z^{n} F\right.$ : $n \geq 0\}$ is all of $H^{p}(\mathbf{D})$.

3. SPECTRAL DeCOMPOSItIONS OF ISOMETRIC GROUPS ON $H^{p}(\mathbf{D}), 1<p<+\infty$

We will introduce two lemmas that will be utilized in the proof of the main theorems to follow in $\S 3$. The first, Lemma (3.1), suggests restricting attention to a special class of $H^{p}(\mathbf{D})$-functions. The second, Lemma (3.2), allows us to assert that the Stone-type spectral family of the parabolic and hyperbolic isometric group on $H^{p}(\mathbf{D})$ (and on $H^{p}\left(\mathbf{T}^{2}\right)$, in $\S 4$ ) is strongly continuous, but it is of independent interest also.

Lemma (3.1). (a) $f_{1}(z)=(1-z)^{1-2 / p}$ is in $H^{p}(\mathbf{D})$ and is outer.

(b) If $Y=\operatorname{sp}\left\{f_{m}(z)=(1-z)^{m-2 / p}, m \geq 1\right\}$ then $\bar{Y}=H^{p}(\mathbf{D})$. 
Proof. (a) Observe that $1-2 / p>-1 / p$ for $p \in(1,+\infty)$ and also that $2 / p-1>-1 / r$ for any $r>0$ if $1<p \leq 2$, and $2 / p-1>-1 / r$ for any $r \in(0, p /(p-2))$ if $2<p<+\infty$. Now apply Lemma (2.4) and Lemma (2.5).

(b) It is immediate from the reasoning in (a) that $f_{m}(z) \in H^{p}(\mathbf{D})$. We can easily also check that $Y=\operatorname{sp}\left\{z^{n} f_{1}: n \geq 0\right\}$ and the result now follows directly by use of Lemma (2.6).

Lemma (3.2). Suppose $\left\{T_{t}\right\}, t \in \mathbf{R}$, is a strongly continuous uniformly bounded one-parameter group on a UMD space $X$, and let $\varepsilon(\cdot)$ be its Stone-type spectral family (as in (1.1)). Suppose also that $G$ denotes the infinitesimal generator of the group and $\sigma_{p}(G)$ the point spectrum of $G$. Then if for some $\lambda \in \mathbf{R}$ we have that $i \lambda \notin \sigma_{p}(G)$ we can conclude that $\varepsilon(\cdot)$ is strongly (left) continuous at $\lambda$.

Remark. It is evident that the above lemma is applicable in particular for $T_{t}=$ $V_{t}$ or $Y_{t}$ on $X=H^{p}(\mathbf{D})$ or $H^{p}\left(\mathbf{T}^{2}\right), 1<p<+\infty$.)

Proof. Suppose $i \lambda \notin \sigma_{p}(G)$ and let $x \in\{\varepsilon(\lambda)-\varepsilon(\lambda-)\} X$. By definition of $\int_{-a}^{a} \lambda d \varepsilon(\lambda)$, as a Stieltjes integral in the strong operator topology, we have that $\int_{-a}^{a} \lambda d \varepsilon(\lambda) x=\lambda x$ for sufficiently large $a>0$. But according to [1, Theorem (4.20] the domain of $G$ is given by $\mathscr{D}_{G}=\left\{x \in X: \lim _{a \rightarrow+\infty} \int_{-a}^{a} \lambda d \varepsilon(\lambda) x\right.$ exists $\}$ and in addition if $x \in \mathscr{D}_{G}$ we have $G x=\lim _{a \rightarrow \infty} \int_{-a}^{a} \lambda d \varepsilon(\lambda) x$. Thus $G x=i \lambda x$ and so $x=0$. In conlcusion $\varepsilon(\lambda)=\varepsilon(\lambda-)$ and since by definition $\varepsilon(\lambda)=\varepsilon(\lambda+)$ the result follows.

We are ready now to present our first theorem, whose results will facilitate the calculations to come in the proof of the second (and main) theorem (Theorem (3.5)).

Theorem (3.3). Let $\left\{V_{t}\right\}$ (resp. $\left.\left\{Y_{t}\right\}\right), t \in \mathbf{R}$, be the normalized parabolic (resp. hyperbolic) isometric group on $H^{p}(\mathbf{D})$. Then

$$
\left.\mathrm{st}_{\varepsilon \rightarrow 0+} \frac{1}{i \pi} \int_{\varepsilon<|t|<1 / \varepsilon} \frac{e^{i \lambda t}}{t} V_{-t} d t \quad \text { (resp. st-lim } \int_{\varepsilon \rightarrow 0+} \frac{e^{i \lambda t}}{t} Y_{-t} d t\right)
$$

exists as an operator $V_{\lambda}$ (resp. $\left.Y_{\lambda}\right)$ in $H^{p}(\mathbf{D})$ and it is involutive. Moreover if $E_{p}(\cdot)$ is the Stone-type spectral family of $\left\{V_{t}\right\}$ (resp. $\left.\left\{Y_{t}\right\}\right), t \in \mathbf{R}$, then

$$
E_{p}(\lambda)=\frac{1}{2}\left(I+V_{\lambda}\right) \quad\left(\text { resp. } F_{p}(\lambda)=\frac{1}{2}\left(I+Y_{\lambda}\right)\right) \quad \text { for any } \lambda \in \mathbf{R} .
$$

Proof. (First notice that in the definitions of $\left\{V_{t}\right\},\left\{Y_{t}\right\}, V_{\lambda}$ and $Y_{\lambda}$ the dependence on $p$ is surpressed for a simpler notation.) We will appeal to the abstract results of [5, Theorem (5.16)] where the existence of such a limit operator, say $T_{\lambda}$, was demonstrated for the case of any strongly continuous one-parameter group of operators that is uniformly bounded on a UMD space. In the same paper if $\varepsilon(\cdot)$ denoted the Stone-type spectral family of this group then

$$
T_{\lambda}=\varepsilon(\lambda)+\varepsilon(\lambda)-I \quad \text { for any } \lambda \in \mathbf{R} \text {. }
$$


It is evident that in particular for the isometric groups we deal with and on $H^{p}(\mathbf{D}), 1<p<+\infty$, we will have automatically

$$
V_{\lambda}=E_{p}(\lambda)+E_{p}(\lambda-)-I \quad\left(\text { resp. } Y_{\lambda}=F_{p}(\lambda)+F_{p}(\lambda-)-I\right) \quad \text { for any } \lambda \in \mathbf{R} \text {. }
$$

But it was shown in [6, Theorem (3.1)] that the infinitesimal generator of $\left\{V_{t}\right\}$ (resp. $\left\{Y_{t}\right\}$ ), $t \in \mathbf{R}$, on $H^{p}(\mathbf{D})$ has no point-spectrum. In light of our Lemma (2.8) we conclude that

$$
E_{p}(\lambda)=E_{p}(\lambda-) \quad\left(\text { resp. } F_{p}(\lambda)=F_{p}(\lambda-)\right) \quad \text { for any } \lambda \in \mathbf{R}
$$

which implies (3.4). But $E_{p}(\lambda)$ (resp. $F_{p}(\lambda)$ ) is a projection on $H^{p}(\mathbf{D})$ for any $\lambda \in \mathbf{R}$ which, by (3.4), implies directly that

$$
V_{\lambda}^{2}=I \quad\left(\text { resp. } Y_{\lambda}^{2}=I\right) \quad \text { for any } \lambda \in \mathbf{R} \text {. }
$$

Remarks. (a) In [16, Theorem 1(ii)], with the aid of contour integration and Lemma (3.1)(b) we were able to show-in a straightforward way-that $\left.V_{\lambda}^{2}\right|_{Y}=$ $\left.I\right|_{Y}$ to conclude that $V_{\lambda}$ is involutive on $H^{p}(\mathbf{D})$ for any $\lambda \in \mathbf{R}$. Then by appealing to the formula $\varepsilon(\lambda)=I+\frac{1}{2}\left(T_{\lambda}-T_{\lambda}^{2}\right), \lambda \in \mathbf{R}$, which is also proved in [5, Theorem 5.12(iii)], we achieved the results of Theorem (3.3) without appealing to Lemma (3.2) and the results in [6, Theorem (3.1)] and [5, Theorem 5.12(ii)]. On the other hand the involutive character of $\left.Y_{\lambda}\right|_{Y}$ in the hyperbolic case, seems much more involved to be demonstrated only within the context of classical contour integration.

(b) The comment in (a) and the fact that the formula $V_{\lambda}=E_{p}(\lambda)+E_{p}(\lambda-)-I$ is guaranteed in advance implies that simply the result $V_{\lambda}^{2}=I$ for any $\lambda \in \mathbf{R}$ leads to the strong (left) continuity of $E_{p}(\lambda)$ on $\mathbf{R}$. This result should be rather expected from the standard theory of the translation groups on $L^{p}(\mathbf{R})$. On the other hand the strong continuity of $F_{p}(\lambda)$ on $\mathbf{R}$ for the reasons that were mentioned before is highly technically involved and other means (e.g. Lemma (3.2) etc.) had to come into play. Still this result is, again, rather natural when viewed in the context of the translation groups theory on $L^{p}\left(\mathbf{R}^{*}\right)\left(\mathbf{R}^{*}\right.$ indicating the multiplicative group of the nonzero reals).

The next theorem, which is rather technical, combining the results of Lemma (3.1)(b) and Theorem (3.3), will furnish many pleasant byproducts concerning $E_{p}(\lambda)$; the contour integration techniques involved in its proof were developed in detail in [16, Theorem (1.1.1)]:

Theorem (3.5). Let $\left\{V_{t}\right\}, t \in \mathbf{R}, V_{\lambda}$ and $E_{p}(\lambda)$ be as defined in Theorem (3.3). Then

(i) $V_{\lambda}=I$ for $\lambda \geq 0$;

(ii) $E_{p}(\lambda)=I$ for $\lambda \geq 0$;

(iii) $\left(E_{p}(\lambda) f_{m}\right)(z)=e^{\lambda} \xi_{\lambda}(z) \omega_{m}(\lambda, z)$ for $\lambda<0, z \in \mathbf{D}$,

where we have set (and standardized for the rest of this paper) $\xi_{\lambda}(z)=e^{\lambda(1+z) /(1-z)}$ 
for $\lambda<0$ and

$$
\omega_{m}(\lambda, z)=\sum_{k=0}^{m-1} \frac{(-2 \lambda)^{k}}{k !} f_{m-k}(z)
$$

for $\lambda<0$ and $m \geq 1$ (and we suppressed, again, the dependence of $\omega_{m}(\lambda, z)$ on $p$ ).

Proof. It is evident that (i) $\Rightarrow$ (ii) by use of (3.4).

(i) We use the contour $\mathscr{C}=\bigcup_{j=1}^{4} l_{j}$, where $l_{1}=[-1 / \varepsilon,-\varepsilon], l_{2}=c(0, \varepsilon)$ (the semicircle lying in the UHP centered at the origin and of radius $\varepsilon), l_{3}=[\varepsilon, 1 / \varepsilon]$ and $l_{4}=c(0,1 / \varepsilon)$. Consider $\mathscr{C}$ travelled c.c.w. We intend to show that $\left.V_{\lambda}\right|_{Y}=\left.I\right|_{Y}$. Then by Lemma $(3.1)(\mathrm{b})$ we have $V_{\lambda}=I$. We need to demonstrate, therefore, that

$$
\left(V_{\lambda} f_{m}\right)(z)=\lim _{\varepsilon \rightarrow 0+} \frac{1}{i \pi} \int_{l_{1} \cup l_{3}} \frac{e^{i t \lambda}}{t}\left(V_{-t} f_{m}\right)(z) d t=f_{m}(z) \quad \text { for } m \geq 1, z \in \mathbf{D} .
$$

But $\left(V_{\lambda} f_{m}\right)(z)=\left\{n_{t}^{\prime}(z)\right\}^{1 / p} f_{m}\left(n_{t}(z)\right)=f_{m}(z) d(t, z)^{-m}$, where $d(t, z)=1+$ $\frac{i}{2}(1-z) t$ is the denominator of $n_{t}(z)$ (see Remark (2) following Proposition (2.2) as well as Lemma (3.4)(a)).

It is easy to check now that in the UHP and its boundary $d(w, z) \neq 0$ $\forall z \in \mathbf{D}$, and thus

$$
\int_{l} \frac{e^{i w \lambda}}{w} d(-w, z)^{-m} d w=0 \text { for } \lambda \geq 0, z \in \mathbf{D}, m \geq 1 .
$$

It can also be checked straightforwardly that

$$
\lim _{\varepsilon \rightarrow 0+} \int_{l_{3}} \frac{e^{i w \lambda}}{w} d(-w, z)^{-m} d w=i \pi \quad \text { and } \quad \lim _{\varepsilon \rightarrow 0+} \int_{l_{4}} \frac{e^{i w \lambda}}{w} d(-w, z)^{-m} d w=0 .
$$

In conclusion $\left(V_{\lambda} f_{m}\right)(z)=f_{m}(z)$ for $\lambda \geq 0, z \in \mathbf{D}$, and $m \geq 1$. Q.E.D.

(iii) In the case $\lambda<0$ we use the symmetric contour of $\mathscr{C}$ w.r.t. the $x$-axis (in the L.H.P.), preserving the notation, as in (i), for $l_{j}, j=1,2,3,4, \mathscr{C}$. This time $\mathscr{C}$ is travelled c.w. and it is clear that $d(-w, z)=0$ has a root $w_{0}=2 / i(1-z)$ in the L.H.P. It can be easily checked that

$$
\lim _{\varepsilon \rightarrow 0+} \int_{l_{3}} \frac{e^{i w \lambda}}{w} d(-w, z)^{-m} d w=i \pi \quad \text { and } \quad \lim _{\varepsilon \rightarrow 0+} \int_{l_{4}} \frac{e^{i w \lambda}}{w} d(-w, z)^{-m} d w=0
$$

for $\lambda<0, z \in \mathbf{D}$, and $m \geq 1$. We conclude that

$$
\left(V_{\lambda} f_{m}\right)(z)=-f_{m}(z)\left\{1+2 \operatorname{Res}\left(w_{0}\right)\right\},
$$

where by $\operatorname{Res}\left(w_{0}\right)$ we have denoted the residue of $\left(e^{i \lambda w} / w\right) d(-w, z)^{-m}$ at $w=w_{0}$. By definition

$$
\operatorname{Res}\left(w_{0}\right)=\left.\frac{1}{(m-1) !} \frac{d^{m-1}}{d w^{m-1}}\left\{\frac{\left(w-w_{0}\right)^{m} e^{i \lambda w}}{w d(-w, z)^{m}}\right\}\right|_{w=w_{0}}=\left[\frac{2 i}{1-z}\right]^{m} a_{m-1}(z),
$$


where $a_{m-1}(z)$ indicates the $(m-1)$ th Taylor coefficient of $e^{i \lambda w} / w$ expanded near $w_{0}$. It is routine to check now that

$$
a_{m-1}(z)=\frac{e^{i \lambda w_{0}}}{w_{0}} \sum_{k=0}^{m-1} \frac{(i \lambda)^{k}}{k !}\left[-\frac{1}{w_{0}}\right]^{m-1-k} .
$$

In conclusion, using (3.4), we have that

$$
\left(E_{p}(\lambda) f_{m}\right)(z)=-f_{m}(z)\left[\frac{2 i}{1-z}\right]^{m} \sum_{k=0}^{m-1} \frac{(i \lambda)^{k}}{k !}\left[-\frac{1}{w_{0}}\right]^{m-1-k}=e^{2 \lambda /(1-z)} \omega_{m}(\lambda, z)
$$

We now have only to observe that $e^{2 \lambda /(1-z)}=e^{\lambda} \xi_{\lambda}(z)$. Q.E.D.

Corollary (3.6). For any $m \geq 1$ and any $\lambda \in(-\infty, \delta]$ where $\delta<-2(m-1)$, the $H^{p}(\mathbf{D})$-functions $E_{p}(\lambda) f_{m}$ have (up to an unimodular constant) as an inner and singular part the function $\xi_{\lambda}(z)$ and as an outer part the function $e^{\lambda} \omega_{m}(\lambda, z)$.

Proof. Using Theorem (3.5)(iii) and the well-known fact that $\xi_{\lambda}(z)$ is inner (and singular following [15]) we have the first part of the stated result. It will suffice therefore to show $\omega_{m}(\lambda, z) \in H^{p}(\mathbf{D})$ and outer. The former is a direct result of Lemma $(3.1)(a)$ and the definition of $\omega_{m}(\lambda, z)$. The latter will be demonstrated if, based on Lemma (2.5), we show that $\omega_{m}(\lambda, z) \neq 0$ in $\mathbf{D}$ and $\omega_{m}(\lambda, z)^{-1} \in H^{r}(\mathbf{D})$ for some $r>0$, whenever $-\infty<\lambda \leq \delta$. Rewrite now $\omega_{m}(\lambda, z)$ as $f_{1}(z) \varphi_{m}(\lambda, z)$,

$$
\varphi_{m}(\lambda, z)=\sum_{k=0}^{m-1} \frac{(-2 \lambda)^{k}}{k !}(1-z)^{m-1-k}
$$

We will show $\varphi_{m}(\lambda, z)$ has no zeros in $\mathbf{D}$ (actually in $\overline{\mathbf{D}}$, whenever $\lambda \in B_{m-1}=$ $(-\infty,-2(m-1))$.

For $m=1$ it is trivially true and we assume thus $m \geq 2$; we appeal to Rouche's theorem using as domain the disk of radius $R, R>1$, and as simple closed curve in this domain the unit circle $\partial \mathbf{D}$. Set $-2 \lambda=\mu>0$ and $|1-z|=a$ and observe that $0 \leq a \leq 2$ on $\partial \mathbf{D}$. By induction it is easy to check the following (stronger) inequality

$$
\sum_{k=0}^{m-2} \frac{\mu^{k}}{k !} a^{m-1-k}<\frac{\mu^{m-1}}{(m-1) !} \quad \text { for } \lambda \in B_{m-1}
$$

(see [16, Corollary (1.1.19)] for details). We can see now that working with $|z|=1+\varepsilon, 0<\varepsilon<R-1$, as the simple closed curve in Rouche's theorem we can derive an analogous result, namely $\varphi_{m}(\lambda, z) \neq 0$ inside $|z|<1+\varepsilon$, for $-\infty<\lambda<-2(m-1)-\tau(\varepsilon)$, where $\tau(\varepsilon) \rightarrow 0^{+}$as $\varepsilon \rightarrow 0^{+}$. (The proof can be repeated verbatim as before, observing that $\varepsilon \leq a \leq 2-\varepsilon$ on $|z|=1+\varepsilon)$. In conclusion $\varphi_{m}(\lambda, z) \neq 0$ in $\overline{\mathbf{D}}$ for $-\infty<\lambda \leq \delta$. Rewrite once more $\omega_{m}(\lambda, z)$ 
as $f_{m}(z) \tilde{\varphi}_{m}(\lambda, z)$ with

$$
\widetilde{\varphi}_{m}(\lambda, z)=\sum_{k=0}^{m-1} \frac{1}{k !}\left[\frac{\mu}{1-z}\right]^{k} .
$$

Evidently $\tilde{\varphi}_{m}(\lambda, z) \neq 0$ in $\mathbf{D}$ for $\lambda \in B_{m-1}$. Notice also that

$$
\lim _{z \rightarrow z_{0}, z \in \mathbf{D}} \tilde{\varphi}_{m}(\lambda, z)^{-1}<+\infty, \quad \text { for any } z_{0} \in \partial \mathbf{D},
$$

and thus $\tilde{\varphi}_{m}(\lambda, z)^{-1} \in H^{\infty}(\overline{\mathbf{D}})$. Hence it will suffice to show that $f_{m}(z)^{-1} \in$ $H^{r}$ (D) for some $r>0$ which, by Lemma (2.4), is now trivial.

Corollary (3.7). For $\lambda<0$ we have $E_{p}(\lambda) H^{p}(\mathbf{D})=\xi_{\lambda}(z) H^{p}(\mathbf{D})$.

Proof. This result was obtained already in [2, Theorem (5.1(i))] by a different approach (use of multiplier operators theory and Fourier transforms on $\left.L^{2}(\mathbf{R})\right)$. We can derive in a straightforward way the same result by means of our representation in (iii) of Theorem (3.5) and by use of Lemma (3.1). It will be enough to show $E_{p}(\lambda) Y=\xi_{\lambda}(z) Y$ for $\lambda<0$. By Theorem (3.5)(iii) we have $E_{p}(\lambda) Y \subset \xi_{\lambda}(z) Y$. To show the reverse inclusion it will suffice to demonstrate $\forall m \in \mathbf{N}$ the existence of a $g(z) \in Y$ (depending on $m$ ): such that $\left(E_{p}(\lambda) g\right)(z)=\xi_{\dot{\lambda}}(z) f_{m}(z), \forall z \in \mathbf{D}$.

We seek thus $b_{1}, b_{2}, \ldots, b_{m} \in \mathbf{C}$ such that

$$
\sum_{n=0}^{m} b_{n}\left(E_{p}(\lambda) f_{n}\right)(z)=\xi_{\lambda}(z) f_{m}(z) \quad \forall z \in \mathbf{D},
$$

which by use of Theorem (3.5)(iii), leads to the system $(*)$

$$
\left\{\begin{array}{r}
b_{1}+\frac{-2 \lambda}{1 !} b_{2}+\cdots+\frac{(-2 \lambda)^{m-1}}{(m-1) !} b_{m}=0 \\
b_{2}+\cdots+\frac{(-2 \lambda)^{m-2}}{(m-2) !} b_{m}=0 \\
e^{\lambda} b_{m}=1
\end{array}\right.
$$

Clearly the determinant of the coefficients is $e^{\lambda} \neq 0$ and we are done.

Concluding remarks on Theorem (3.5). (1) Since

$$
\sum_{k=0}^{m-1} \frac{1}{k !}\left[\frac{-2 \lambda}{1-z}\right]^{k} \rightarrow\left\{e^{\lambda} \xi_{\lambda}(z)\right\}^{-1} \quad \text { as } m \rightarrow+\infty,
$$

we can conclude $\left(E_{p}(\lambda) f_{m}\right)(z) / f_{m}(z) \rightarrow 1$ as $m \rightarrow+\infty$, uniformly on compact subsets of $\mathbf{D}$, for any fixed $\lambda \in \mathbf{R}$. Combining this remark with the normdenseness of $Y$ one could determine the behavior of $E_{p}(\lambda)$ when it acts upon any $H^{p}(\mathbf{D})$-function which is sufficiently "close" to a "large" degree polynomial in $z \in \mathbf{D}$.

(2) Using Theorem (3.5) in the Hilbert space case of $p=2$, we can recapture some known results about the action of $E_{2}(\lambda)$ upon the $H^{2}$ functions (see e.g. 
[22, Theorem (1)]). In addition, for the standard basis $\left\{z^{n}: n \geq 0\right\}$ one has for $\lambda<0$.

$$
\begin{aligned}
E_{2}(\lambda) z^{n} & =E_{2}(\lambda) \sum_{k=0}^{n}(-1)^{k}\left[\begin{array}{l}
n \\
k
\end{array}\right](1-z)^{k} \\
& =e^{\lambda} \xi_{\lambda}(z) \sum_{k=0}^{n}(-1)^{k}\left[\begin{array}{l}
n \\
k
\end{array}\right] \sum_{j=0}^{n} \frac{(-2 \lambda)^{j}}{j !}(1-z)^{k-j} .
\end{aligned}
$$

We can thus have a $\left[\right.$ mean $\left.^{2}\right]$ description of $\left(E_{2}(\lambda) f\right)(z)$ for any $f \in H^{2}(\mathbf{D})$ if we are given its Taylor expansion around $z=0$.

It was shown in [2] that under the natural isometry between $H^{p}(\mathbf{D})$ and $H^{p}(\mathbf{R})$ the projections $E_{p}(\lambda)$ correspond to the partial sum-operators of $\mathrm{M}$. Riesz restricted from $L^{p}(\mathbf{R})$ to $H^{p}(\mathbf{R})$. The next theorem will provide the $H^{p}(\mathbf{D})$ version, for $1<p \leq 2$, of these partial sum-operators:

Theorem (3.8). For $\lambda<0$ and $1<p \leq 2$ we have that

$$
E_{p}(\lambda)(1-z)^{m}=e^{\lambda} \xi_{\lambda}(z) \sum_{n=0}^{+\infty} c_{n} \sum_{k=0}^{n}(-1)^{k}\left[\begin{array}{l}
n \\
k
\end{array}\right] \omega_{m+1+k}(\lambda, z) \quad\left[\text { in mean }^{p}\right],
$$

where $m \geq 0, c_{0}=1, c_{n}=a(a+1) \cdots(a+n-1) / n$ ! for $n \geq 1$ and $a=1-2 / p$. Proof. If $p=2$ the above formula agrees with the formula in the latest Remark (2). We therefore restrict oursleves to $p \in(1,2)$. (Notice also that for $p \neq 2, \lambda<0$, the elementary residual approach of Theorem (3.5) is inadequate since the integrands involved there have a singularity of noninteger order in the LHP.) We first realize $(1-z)^{m}$ as an $H^{p}$-limit of elements in $Y$. We write $(1-z)^{m}=f_{m+1}(z) f_{1}(z)^{-1}$. We know already that $f_{m+1}(z) \in H^{p}(\mathbf{D})$ and we can easily check that $f_{1}(z)^{-1}$ is analytic in $\mathbf{D}$ and its derivative, $(1-2 / p) f_{2}(z)^{-1}$, is in $H^{1}$ (D) by Lemma (2.4). By Theorem (3.11) in [12] we conclude that $f_{1}(z)^{-1}$ is continuous on $\overline{\mathbf{D}}$ and absolutely continuous on $\partial \mathbf{D}$. In particular $f_{1}(z)^{-1}$ is continuous and of bounded variation on $\partial \mathbf{D}$. By Fejer's theorem (see e.g. [17]) and a Tauberian theorem of Hardy [17, II.2.2] we conclude that the Fourier series of $f_{2}(z)^{-1}$ converges uniformly to it on $\partial \mathbf{D}$ (in fact absolutely on $\partial \mathbf{D}$ by virture of Hardy's inequality, see e.g. [12, p. 48]. But the Fourier coefficients of the boundary function $f_{1}\left(e^{i \theta}\right)^{-1}$ coincide with the Taylor coefficients of $f_{1}(z)^{-1}$ and are easily found to be $c_{0}=1$ and $c_{n}=(a(a+1) \cdots(a+n-1)) / n$ ! for $n \geq 1$ with $a=1-2 / p$. Thus the partial sums $\sum_{n=0}^{N} c_{n} z^{n}$ converge uniformly on $\partial \mathbf{D}$ to $f_{1}(z)^{-1}$, as $N \rightarrow+\infty$, and therefore multiplication by $f_{m+1}(z)$ provides [mean ${ }^{p}$ ] convergence.

Applying now the projection $E_{p}(\lambda), \lambda>0$, termwise and factoring out $e^{\lambda} \xi_{\lambda}(z)$ (which does not alter the convergence since $\xi_{\lambda}(z)$ is inner) we arrive after simple manipulations, by use of the formula (iii) of Theorem (3.5), at the stated result. 
Remarks. (1) It is immediate now that the concluding remark (2) on Theorem (3.5) can be extended to any $H^{p}(\mathbf{D})$ function for $1<p \leq 2$.

(2) The method of the proof of Theorem (3.8) is evidently not applicable for $1<p<+\infty$. We can however capture the action of $E_{p}(\lambda), \lambda<0$, on the constant functions in $H^{p}(\mathbf{D})$, even for the unnatural range $2<p<3$, by writing $1 \equiv f_{1}(z)^{-1} f_{1}(z)$; the left factor is in $H^{p}(\mathbf{D})$ and the right factor has an $H^{1}(\mathbf{D})$ derivative. Thus we only need now to set $m=0$ in the proof of Theorem (3.8) to find $\left(E_{p}(\lambda) e_{0}\right)(z), e_{0}(z)=1$. (It seems as a rather interesting question extending Theorem (3.8) to the whole range $1<p<+\infty$.)

We conclude this section with an application of Theorem (3.8) in the case of the "F. Riesz-B. Nagy operators", $Q_{n}$, (defined below) on $H^{p}(\mathbf{D})$; we need first to present some concepts and facts that we use later in Corollary (3.10).

Definition. An operator $U$ on a Banach space $X$ is called trigonometrically well-bounded if it can be expressed by

$$
U=\varepsilon(0)+\int_{[0,2 \pi]} e^{i \lambda} d \varepsilon(\lambda) \quad \text { (as strong limit of Stieltjes sums), }
$$

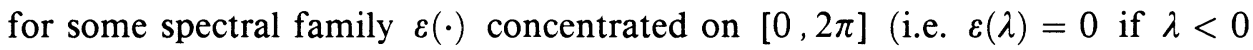
and $\varepsilon(\lambda)=I$ if $\lambda \geq 2 \pi)$.

We can always arrange $\varepsilon((2 \pi)-)=I$ and then $\varepsilon(\cdot)$ is uniquely determined.

Definition. An operator $U$ on $X$ is called power-bounded if it invertible and $\sup \left\{\left\|U^{n}\right\|: n \in \mathbf{Z}\right\}<+\infty$.

A very crucial fact about a power-bounded operator $U$ on a UMD space is that any such operator is trigonometrically well-bounded [5, Theorem (4.5)]. In particular in our context of surjective isometries on $H^{p}(\mathbf{D})$ (and on $H^{p}\left(\mathbf{T}^{2}\right)$ as well) it is automatic that we deal with trigonometrically well-bounded operators. We thus can formulate in our context all the results concerning trigonometrically well-bounded operators of [4], and in particular the result of [4, Proposition $(2.15)$ ] as follows:

Lemma (3.9). If $U$ is a surjective isometry on $H^{p}(\mathbf{D}), 1<p+\infty$, then there is a unique (bounded) operator in $H^{p}(\mathbf{D}), A$, such that $U=e^{i A}, \sigma(A) \subset[0,2 \pi]$ and $2 \pi \notin \sigma_{p}(A)$. (In fact $A$ is a well-bounded operator of type $(B)$, that is, there is some compact interval $[a, b]$ and a (unique) spectral family $\varepsilon(\cdot)$ in $H^{p}(\mathbf{D})$ concentrated on $[a, b]$, such that $A=a \varepsilon(a)+\int_{[a, b]} \lambda d \varepsilon(\lambda)$.)

Definition. We define the F. Riesz-B. Nagy operators $Q_{n}, n \in \mathbf{Z}$, in $H^{p}(\mathbf{D})$ by

$$
Q_{n}=\int_{0}^{1} e^{2 \pi i n t} V_{t} e^{-i t A_{1}} d t
$$

(as strong limit of Riemann sums), where $\left\{V_{t}\right\}, t \in \mathbf{R}$, is the standard parabolic isometric group on $H^{p}(\mathbf{D})$, and $A_{1}$ is the "normalized" logarithm of $V_{1}$ as defined in Lemma (3.9). 
Remark. The operators $Q_{n}$ exist in a more general Banach space setting (see e.g. $[1,(4.20)$ or 20$])$.

Corollary (3.10). For the operators $Q_{n}, n \in \mathbf{Z}$, as defined above, we have

(i) $Q_{n}=0$ for $n \leq 0$;

(ii) $Q_{n}=E_{p}(-2 \pi(n-1))-E_{p}(2 \pi n)$ for $n \geq 1$, where $E_{p}(\cdot)$ is the Stone-type spectral family of $\left\{V_{t}\right\}, t \in \mathbf{R}$.

Proof. (i) According to (3.5), in [2] for all $\lambda \in \mathbf{R}$ we have

$$
E_{p}(2 \pi \lambda)=\sum_{n=-\infty}^{[\lambda]-1} P_{n}+P_{[\lambda]} \varepsilon_{1}(2 \pi(\lambda-[\lambda]))
$$

where $P_{n}=Q_{-n}, \varepsilon_{1}(\cdot)$ is the spectral family of $A_{1}$ and $[\lambda]$ indicates the integer-part of $\lambda$. But it was also shown in [16, Lemma (II, 1.5) and Remark (2)] that if $\varepsilon_{t_{0}}(\cdot)$ denotes the spectral family of $A_{t_{0}}$, the "normalized" logarithm of $V_{t_{0}}$ (presented in Lemma (3.9)), then $\varepsilon_{t_{0}}(\lambda)=\varepsilon_{t_{0}}(\lambda-)$ for $0 \leq \lambda<2 \pi$. In particular for $\lambda=0$ and $t_{0}=1$ we have $\varepsilon_{1}(0)=\varepsilon_{1}(0-)=\lim _{\lambda \rightarrow 0, \lambda<0} \varepsilon_{1}(\lambda)=0$ since $\varepsilon_{1}(\cdot)$ is concentrated on $[0,2 \pi]$. By Theorem $(3.5)(\mathrm{ii}) E_{p}(0)=I$ and thus (3.11) reduces to $I=\sum_{n=-\infty}^{-1} P_{n}$ (all these sums are interpreted in the strong operator topology). But from the general theory $\sum_{n=-\infty}^{+\infty} P_{n}=I$ and thus we conclude $\sum_{n=0}^{+\infty} P_{n}=0$. Since in general $P_{m}, m \in \mathbf{Z}$, are projections such that $P_{m} P_{n}=P_{n} P_{m}=0$ for $m \neq n$, we automatically have $P_{n}=0$ for $n \geq 0$.

(ii) Using again (3.11) for $\lambda=-1,-2, \ldots,-n$ we see, after "telescoping",

$$
Q_{n}=P_{-n}=E_{p}(-2 \pi(n-1))-E_{p}(-2 \pi n), \quad n \geq 1 .
$$

(Notice that (ii) is trivially true for $n \leq 0$ also, by (i) and Theorem (3.5)(ii) combined.)

Remark. In light of our results in Theorem (3.5) and Theorem (3.8) we see now that we can describe concretely $\left.Q_{n}\right|_{Y}$, and in particular for $1<p \leq 2$ we can describe $\left(Q_{n} f\right)(z) \in H^{p}(\mathbf{D})$, for any $n \in \mathbf{Z}$, if we are given the Taylor coefficients (around $z=0$ ) of $f(z) \in H^{p}(\mathbf{D})$.

4. SPeCtral deCOMPOSITIONS OF ISOMETRIC GROUPS ON $H^{p}\left(\mathbf{T}^{2}\right), 1<p<+\infty$

Definition (4.1). $H^{p}\left(\mathbf{T}^{2}\right)$ is defined as the subspace of complex $L^{p}\left(\mathbf{T}^{2}\right)$ consisting of those functions whose double Fourier coefficients $c_{k j}$ vanish if $(k, j) \notin$ $\{(m, n): n \geq 1\} \cup\{(m, 0): m \geq 0\}$.

Definition (4.2). We denote by $Z^{p}$ the closure of the polynomials in $z$, by $\Lambda_{1}^{p}$ the closure of the polynomials in $z_{1}^{m}, z_{2}^{n}, m \geq 0$ and $n \geq 1$, and by $\Lambda_{2}^{p}$ the closure of the polynomials in $z_{1}^{-m} z_{2}^{n}, m \geq 1, n \geq 1$, all considered in $L^{p}(d \mu)$, where $d \mu$ is the normalized Haar measure on the torus $\mathbf{T}^{2}$. 
Let now $\Omega_{p}$ be the set of all strongly continuous one-parameter group of isometries on $H^{p}\left(\mathbf{T}^{2}\right)$ such that $\left\{\left.T_{t}\right|_{Z^{p}}\right\}, t \in \mathbf{R}$, is not continuous in the uniform operator topology. (This last stipulation on $\left\{T_{t}\right\}$ is necessary to us in order to avoid descriptions widely different from the ones already used on $H^{p}(\mathbf{D})$ in $\S 2$.) In case $p=2$ we require, in addition, that each $T_{t}$ has the representation

$$
(T f)\left(z_{1}, z_{2}\right)=a\left\{\varphi^{\prime}\left(z_{1}\right)\right\}^{1 / 2} f\left(\varphi\left(z_{1}\right), \sigma\left(z_{1}\right) z_{2}\right) \quad \text { for a.a. }\left(z_{1}, z_{2}\right) \in \mathbf{T}^{2},
$$

where $a \in \mathbf{T}, \varphi$ is a Möbius transformation of $\mathbf{D}$ and $\sigma: \mathbf{T} \rightarrow \mathbf{T}$ is measurable (see [8] for details).

The next theorem combines results contained in [8].

Theorem. Let $\left\{T_{t}\right\} \in \Omega_{p}$. Then there are $\rho \in \mathbf{R},\left\{\varphi_{t}\right\}, t \in \mathbf{R}$, a one-parameter group of Möbius transformations of $\mathbf{D}$, and a one-parameter family $\left\{\sigma_{t}\right\}, t \in$ $\mathbf{R}$, of unimodular measurable functions on $\mathbf{T}$ (with each $\sigma_{t}$ determined up to equality a.e.) such that

(i) $\left(T_{t} f\right)\left(z_{1}, z_{2}\right)=e^{i \rho t}\left\{\varphi_{t}^{\prime}\left(z_{1}\right)\right\}^{1 / p} f\left(\varphi_{t}\left(z_{1}\right), \sigma_{t}\left(z_{1}\right) z_{2}\right)$, for $t \in \mathbf{R}, f \in H^{p}\left(\mathbf{T}^{2}\right)$ and a.a. $\left(z_{1}, z_{2}\right) \in \mathbf{T}^{2}$;

(ii) $\sigma_{t+s}(z)=\sigma_{t}\left(\varphi_{s}(z)\right) \sigma_{s}(z)$ for $t, s \in \mathbf{R}$ and a.a. $z \in \mathbf{T}$;

(iii) $t \rightarrow \sigma_{t}$ is continuous $\mathbf{R} \rightarrow L^{p}(\mathbf{T})$.

Conversely if $\left\{\sigma_{t}\right\}$ is defined as above (and (ii) and (iii) hold) and $\rho \in \mathbf{R}$ and $\left\{\varphi_{t}\right\}, t \in \mathbf{R}$, is a Möbius group on $\mathbf{D}$ then (i) defines a group $\left\{T_{t}\right\} \in \Omega_{p}$.

We will call $\left\{T_{t}\right\} \in \Omega_{p}$ parabolic or hyperbolic according to the corresponding classification of $\left\{\varphi_{t}\right\}, t \in \mathbf{R}$, given in Proposition (2.1).

Remarks. (1) In our calculations for reasons that later become clear we can assume WLOG $\rho=0$. As it was shown in [8, Theorem (2.6)] for each $\left\{T_{t}\right\} \in$ $\Omega_{p}$ we have a unimodular measurable function $u: \mathbf{T} \rightarrow \mathbf{T}$ such that, for each $t \in \mathbf{R}, \sigma_{t}(z)=u\left(\varphi_{t}(z)\right) \bar{u}(z)$ for a.a. $a \in \mathbf{T}$.

(2) Following [8], if $U$ indicates the isometry on $H^{p}\left(\mathbf{T}^{2}\right)$ given by

$$
(U f)\left(z_{1}, z_{2}\right)=f\left(z_{1}, u\left(z_{1}\right) z_{2}\right)
$$

and if we set $S_{t}=U T_{t} U^{-1}$ then

$$
\left(S_{t} f\left(z_{1}, z_{2}\right)\right)=\left\{\varphi_{t}^{\prime}\left(z_{1}\right)\right\}^{1 / p} f\left(\varphi_{t}\left(z_{1}\right), z_{2}\right)
$$

for $f \in H^{p}\left(\mathbf{T}^{2}\right)$ and a.a. $\left(z_{1}, z_{2}\right) \in \mathbf{T}^{2}$. In conclusion the study of $\left\{T_{t}\right\}, t \in \mathbf{R}$, reduces to the study of $\left\{S_{t}\right\}, t \in \mathbf{R}$, and working as in Lemma (2.3) we can consider WLOG the conformal group $\left\{\varphi_{t}\right\}$ to be $\left\{n_{t}\right\}$ (resp. $\left\{\psi_{t}\right\}$ ), $t \in \mathbf{R}$, for the parabolic (resp. hyperbolic) case. We will denote the corresponding group by $\left\{V_{\tau}\right\}$ (resp. $\left.\left\{{\underset{\sim}{Y}}_{t}\right\}\right), t \in \mathbf{R}$. Thus we will have always the following pointwise description:

$$
\left(\underset{\sim}{V_{t}} f\right)\left(z_{1}, z_{2}\right)=\left\{n_{t}^{\prime}\left(z_{1}, z_{2}\right)\right\}^{1 / p} f\left(n_{t}\left(z_{1}\right), z_{2}\right)
$$


(resp. $\left.\left(\underset{\sim}{Y_{t}} f\right)\left(z_{1}, z_{2}\right)=\left\{\psi_{t}^{\prime}\left(z_{1}, z_{2}\right)\right\}^{1 / p} f\left(\psi_{t}\left(z_{1}\right), z_{2}\right)\right)$, for $f \in H^{p}\left(\mathbf{T}^{2}\right)$ and a.a. $\left(z_{1}, z_{2}\right) \in \mathbf{T}^{2}$.

From now on we will also fix the notation $\underset{\sim}{E_{p}}(\cdot)\left(\right.$ resp. $\left.\underset{\sim}{F_{p}}(\cdot)\right)$ to indicate the Stone-type spectral family of $\left\{\underset{\sim}{V_{t}}\right\}$ (resp. $\left.\left\{\underset{\sim}{Y_{t}}\right\}\right), t \in \mathbf{R}$, and $\underset{\sim}{V_{\lambda}}$ (resp. $\underset{\sim}{Y_{\lambda}}$ ), $\lambda \in \mathbf{R}$, to indicate the corresponding limit-operator as defined in Theorem (3.3); the existence of $V_{\lambda}$ (resp. ${\underset{\sim}{Y}}_{\lambda}$ ) in $H^{p}\left(\mathbf{T}^{2}\right)$ has been already discussed in a more general setting on a UMD space and the formulae, the corresponding to the ones used in the proof of Theorem (3.3), in this new context are given by

$$
\begin{aligned}
& \underset{\sim}{V_{\lambda}}=\underset{\sim}{E_{p}}(\lambda)+\underset{\sim}{E_{p}}(\lambda-)-I \quad\left(\text { resp. } \underset{\sim}{Y}{ }_{\lambda}=\underset{\sim}{F_{p}}(\lambda)+\underset{\sim}{F_{p}}(\lambda-)-I\right) \text {, } \\
& \underset{\sim p}{E_{p}}(\lambda)=I+\frac{1}{2}\left(\underset{\sim}{V_{\lambda}}-\underset{\sim}{V_{\lambda}^{2}}\right) \quad\left(\operatorname{resp} . \underset{\sim p}{F_{p}}(\lambda)=I+\frac{1}{2}\left(\underset{\sim}{Y_{\lambda}}-\underset{\sim}{Y_{\lambda}^{2}}\right)\right)
\end{aligned}
$$

for all $\lambda \in \mathbf{R}$. (Once again the dependence of $\underset{\sim}{V}$ (resp. $\underset{\sim}{Y_{\lambda}}$ ) on $p$ was supressed for notational convenience.)

It is important, at this point, to note that the lexicographic order of the $(m, n)$-half plane in Definition (4.1) is not symmeetric in $\left(z_{1}, z_{2}\right)$. This lack of symmetry does not allow a verbatim reproduction of the results of Theorem (3.5). On the other hand in [16, Lemma (III, 1.2) and Theorem (III, 1.23)], employing (4.4) and contour integration, we were able to demonstrate the involutive character of $\underset{\sim}{V}$ for $\lambda \geq 0$ and to study the Hilbert case $\underset{\sim}{E_{2}}(\lambda) H^{2}\left(\mathbf{T}^{2}\right)$ for $\lambda \in \mathbf{R}$. (The use of (4.4) besides the many technicalities that it produced, required at some stage extending - in the standard way $-V_{t}$ and ${\underset{\sim}{\lambda}}_{\lambda}$ on $L^{p}\left(\mathbf{T}^{2}\right)$.) We can generalize the above results of [16] by appealing to (4.3) and Lemma (3.2), after we introduce the following two lemmas; the first will provide us with a suitable "decomposition" of $H^{p}\left(\mathbf{T}^{2}\right)$ (the analog to Lemma $(3.1)$ (b) for $\left.H^{p}(\mathbf{D})\right)$, and the second will guarantee the (left) strong continuity of $\underset{\sim}{E_{p}}(\lambda)$ and $\underset{\sim p}{F_{p}}(\lambda)$ for $\lambda \in \mathbf{R}$, a result which will make (4.3) easy to utilize.

Lemma (4.5). For $Z^{p}, \bigwedge_{1}^{p}, \bigwedge_{2}^{p}$ the subspaces of $L^{p}(d \mu)$ defined in (4.2) we have $H^{p}\left(\mathbf{T}^{2}\right)=Z^{p} \oplus \bigwedge_{1}^{p} \oplus \bigwedge_{2}^{p}$, where $\oplus$ indicates direct sum.

Proof. In [19] it was proved that $H^{p}\left(\mathbf{T}^{2}\right)=Z^{p} \oplus \bigwedge^{p}$ was the $L^{p}(d \mu)$-closure of $\operatorname{sp}\left\{z_{1}^{m} z_{2}^{n}, m \in \mathbf{Z}, n \in \mathbf{N}\right\}$. It is evident now that $\operatorname{sp}\left\{z_{1}^{m} z_{2}^{n}, m \in \mathbf{Z}, n \in \mathbf{N}\right\}=$ $S_{1}^{p} \oplus S_{2}^{p}$ where $S_{1}^{p}=\operatorname{sp}\left\{z_{1}^{m} z_{2}^{n}, m \in \mathbf{Z}, n \in \mathbf{N}\right\}$ and $S_{2}^{p}=\operatorname{sp}\left\{z_{1}^{-m} z_{2}^{n}, m, n, \in\right.$ $\mathbf{N}\}$. If we use the left to right lexicographic ordering of $\mathbf{Z} \times \mathbf{Z}$ the $S_{1}^{p}, S_{2}^{p}$ are spans of positive characters. It follows from Bochner's generalization of M. Riesz's theorem on conjugate harmonic functions (see e.g. [3]) that $\Lambda^{p} \subset$ $\bigwedge_{1}^{p} \oplus \bigwedge_{2}^{p} \subset H^{p}\left(\mathbf{T}^{2}\right)$ and thus $\bigwedge^{p}=\bigwedge_{1}^{p} \oplus \bigwedge_{2}^{p}$. Q.E.D.

Remark. It is evident that $Z^{p}$ can be "identified" with $H^{p}(\mathbf{T})$ so that, by Lemma (4.5), $H^{p}\left(\mathbf{T}^{2}\right)$ can be "identified" with $H^{p}(\mathbf{T}) \oplus \bigwedge_{1}^{p} \oplus \bigwedge_{2}^{p}$. 
Lemma (4.6). Let $\left\{{\underset{\sim}{t}}_{t}\right\}$ (resp. $\left.\left\{\sim_{t}^{Y}\right\}\right)$ be the standard parabolic (resp. hyperbolic) isometric group on $H^{p}\left(\mathbf{T}^{2}\right), 1<p<+\infty$. Then the infinitesimal generator $G$ of $\left\{V_{t}\right\}$ (resp. $\left.\left\{{\underset{\sim}{t}}_{t}\right\}\right)$ has no point-spectrum.

Proof. Since $\left\{V_{t}{ }_{\tau}\right\}$ and $\left\{{\underset{\sim}{t}}_{t}\right\}$ are uniformly bounded groups then $\sigma_{p}(G) \subset i \mathbf{R}$, for each case (see e.g. [10]).

(i) For the parabolic case we know that

$$
\left(V_{\sim} f\right)\left(z_{1}, z_{2}\right)=\left\{n_{t}^{\prime}\left(z_{1}\right)\right\}^{1 / p} f\left(n_{t}\left(z_{1}\right) z_{2}\right)
$$

for $f \in H^{p}\left(\mathbf{T}^{2}\right)$ and a.a. $\left(z_{1}, z_{2}\right) \in \mathbf{T}^{2}$. Then if $\lambda \in \mathbf{R}$ and $f\left(e^{i \theta}, z_{2}\right)$ is in the null-space of $G-i \lambda I$ we have, by [8, Theorem (3.17)], for a.a. $z_{2} \in \mathbf{T}$

$$
i \lambda f\left(e^{i \theta}, z_{2}\right)=-i e^{i \theta} q\left(e^{i \theta}\right) \frac{d}{d \theta} f\left(e^{i \theta}, z_{2}\right)+\frac{1}{p} q^{\prime}\left(e^{i \theta} f\left(e^{i \theta}, z_{2}\right),\right.
$$

for a.a. $z_{2}=e^{i \theta} \in \mathbf{T}$. In our case it is easy to check that $q(z)=\frac{i}{2}(z-1)^{2}$ for $z \in \mathbf{C}$ and that for a.a. $z_{2} \in \mathbf{T}$ (4.7) holds for a.a. $z_{1} \in \mathbf{T}$ when $f\left(e^{i \theta}, z_{2}\right)$ is absolutely continuous on each closed subinterval of $(0,2 \pi)$. Notice also that for any such $z_{2}$ we have on each $[\varepsilon, 2 \pi-\varepsilon], \varepsilon>0$ sufficiently small, a continuous function that equals $(d / d \theta) f\left(e^{i \theta}, z_{2}\right)$ for a.a. $\theta$. Hence for such a $z_{2}$ the absolute continuity in $\theta$ of $f\left(e^{i \theta}, z_{2}\right)$ implies that (4.7) holds for all $\theta \in(0,2 \pi)$.

Following an idea from [6, Theorem (2.7)], we seek an integrating factor for (4.7) as follows: let $F\left(z_{1}\right)=\left(z_{1}-1\right)^{2(1 / p-1)} e^{\lambda /\left(z_{1}-1\right)}$ on $\mathbf{T}-\{1\}$. Thus $F\left(z_{1}\right) q\left(z_{1}\right)=\frac{i}{2}\left(z_{1}-1\right)^{2 / p} e^{2 \lambda /\left(1-z_{1}\right)}$ for $z_{1} \in \mathbf{T} \backslash\{1\}$ and after multiplying (4.7) by $F$ it is straightforward to verify that, for fixed $z_{2} \in \mathbf{T}$

$$
-i e^{-i \theta} \frac{d}{d \theta}(F q f)=0 \text {. }
$$

Thus $F q f=$ constant depending on $z_{2}$, i.e. $f\left(z_{1}, z_{2}\right)=q\left(z_{2}\right) / F\left(z_{1}\right) q\left(z_{1}\right)$. But $f \in L^{p}\left(\mathbf{T}^{2}\right)$ and so far a.a. $z_{2} \in \mathbf{T}$ we have that

$$
\frac{g\left(z_{2}\right)}{F\left(z_{1}\right) q\left(z_{1}\right)} \in L^{p}\left(\frac{d \theta}{2 \pi}\right) \text {. }
$$

On the other hand $\left|1 / f\left(z_{1}\right) q\left(z_{1}\right)\right|=2 e^{\lambda}\left|1-z_{1}\right|^{-2 / p} \notin L^{p}(d \theta / 2 \pi)$, hence $g\left(z_{2}\right)=0$ for a.a. $a_{2} \in \mathbf{T}$. This shows $f=0$ for a.a. $\left(z_{1}, z_{2}\right) \in \mathbf{T}^{2}$.

(ii) For the hyperbolic group $\left\{{\underset{\sim}{Y}}_{t}\right\}$, associated with

$$
\psi_{t}\left(z_{1}\right)=\left(e^{t}-\left(1-z_{1}\right) /\left(1+z_{1}\right)\right) /\left(e^{t}-\left(1-z_{1}\right) /\left(1+z_{1}\right)\right) .
$$

and for $f\left(e^{i \theta}, z_{2}\right)$ an eigenfunction in $H^{p}\left(\mathbf{T}^{2}\right)$ of $G$ we have, again by [8, Theorem (3.17)], for a.a. $z_{2} \in \mathbf{T}$

$$
-i e^{i \theta} q\left(e^{i \theta}\right) \frac{d}{d \theta} f\left(e^{i \theta}, z_{2}\right)+\frac{1}{p} q^{\prime}\left(e^{i \theta}, z_{2}\right)=i \lambda f\left(e^{i, \theta}, z_{2}\right),
$$


for all $\theta \in[\varepsilon, \pi-\varepsilon] \cup[\pi+\varepsilon, 2 \pi-\varepsilon], \varepsilon>0$, sufficiently small. Here we can easily check that $q(z)=\frac{1}{2}\left(1-z^{2}\right)$ for $z \in \mathbf{C}$. We now take

$$
F\left(z_{1}\right)=\left(z_{1}+1\right)^{1 / p-i \lambda-1}\left(z_{1}-1\right)^{1 / p+i \lambda-1}
$$

for $z_{1} \in \mathbf{T} \backslash\{-1,1\}$. Working with (4.8) as with (4.7), we obtain $g_{1}\left(z_{2}\right)$ (resp. $\left.g_{2}\left(z_{2}\right)\right)$ on $\mathbf{T}$ such that for a.a. $z_{2} \in \mathbf{T}$

$$
f\left(z_{1}, z_{2}\right)=\frac{g_{1}\left(z_{2}\right)}{F\left(z_{1}\right) q\left(z_{1}\right)} \quad\left(\text { resp. } f\left(z_{1}, z_{2}\right)=\frac{g_{2}\left(z_{2}\right)}{F\left(z_{1}\right) q\left(z_{1}\right)}\right) \quad \text { for } z_{1}=e^{i \theta}
$$

with $0<\theta<\pi$ (resp. $\pi<\theta<2 \pi$ ). But here

$$
F\left(z_{1}\right) q\left(z_{1}\right)=-\frac{1}{2}\left(z_{1}+1\right)^{1 / p-i \lambda}\left(z_{1}-1\right)^{1 / p+i \lambda}
$$

and thus we have

$$
\left|\frac{1}{F\left(z_{1}\right) q\left(z_{1}\right)}\right| \geq c\left|z_{1}+1\right|^{1 / p}\left|z_{1}-1\right|^{1 / p},
$$

where $c>0$, so that $\left|1 / F\left(z_{1}\right) q\left(z_{1}\right)\right| \notin L^{p}(d \theta / 2 \pi)$ and thus $1 / F\left(z_{1}\right) q\left(z_{1}\right) \notin$ $L^{p}((0, \pi))$ (resp. $\left.\notin L^{p}((\pi, 2 \pi))\right)$. It follows that $g_{1}\left(z_{2}\right)=0$ (and $g_{2}\left(z_{2}\right)=0$ ) for a.a. $z_{2} \in \mathbf{T}$. Hence $f\left(z_{1}, z_{2}\right)=0$ for a.a. $\left(z_{1}, z_{2}\right) \in \mathbf{T}^{2}$. Q.E.D.

We are ready now to state the main theorem of this section:

Theorem (4.9). Let $\left\{\underset{\sim}{V_{t}}\right\}$ (resp. $\left\{{\underset{\sim}{Y}}_{t}\right\}$ ), $t \in \mathbf{R}$, be the standard parabolic (resp. hyperbolic) isometric group on $H^{p}\left(\mathbf{T}^{2}\right), 1<p<+\infty$. Then

(i) The Stone-type spectral family $\underset{\sim}{E_{p}}(\lambda)$ (resp. $\left.\underset{\sim p}{F_{p}}(\lambda)\right)$ of $\left\{\underset{\sim}{V_{t}}\right\}$ (resp. $\left.\left\{\underset{\sim}{Y_{t}}\right\}\right)$ is strongly continuous on $\mathbf{R}$

(ii) $\underset{\sim}{E}(\lambda)=\frac{1}{2}(I+\underset{\sim}{V})\left(\right.$ resp. $\left.\underset{\sim}{F}(\lambda)=\frac{1}{2}\left(I+Y_{\lambda}\right)\right)$ for any $\lambda \in \mathbf{R}$;

(iii) $V_{\lambda}$ (resp. ${\underset{\sim}{Y}}_{\lambda}$ ) in involutive in $H^{p}\left(\mathbf{T}^{2}\right)$ for any $\lambda \in \mathbf{R}$ and any $p \in$ $(1,+\infty)$

Proof. (i) Combine Lemma (3.2) and Lemma (4.6) to get $\underset{\sim}{E_{p}}(\lambda)=\underset{\sim}{E_{p}}(\lambda-)$ (resp. $\left.\underset{\sim}{F_{p}}(\lambda)=\underset{\sim}{F_{p}}(\lambda-)\right)$ for any $\lambda \in \mathbf{R}$. By definition $\underset{\sim}{E_{p}}(\lambda)=\underset{\sim}{E_{p}}(\lambda+)$ (resp. $\underset{\sim p}{F_{p}}(\lambda)=\underset{\sim}{F_{p}}\left(\lambda_{+}\right)$for any $\lambda \in \mathbf{R}$. Q.E.D.

(ii) Use (i) combined with (4.3).

(iii) Combine (ii) and the fact that $\underset{\sim}{E_{p}}(\lambda)$ (resp. $\underset{\sim}{F_{p}}(\lambda)$ ) is a projection in $H^{p}\left(\mathbf{T}^{2}\right)$, for any $\lambda \in \mathbf{R}$, to get ${\underset{\sim}{\lambda}}_{\lambda}^{2}=I$ (resp. $\underset{\sim}{Y_{\lambda}^{2}}=I$ ) for any $\lambda \in \mathbf{R}$.

Remarks. As mentioned before in an elementary way [16] we have demonstrated the involutive character of $V_{\lambda}$ for $\lambda \geq 0$. Working in a similar fashion and by use of elementary contour integration techniques we could arrive at the same result for $\lambda<0$. Then the fact that $V_{\lambda}^{2}=I$ for any $\lambda \in \mathbf{R}$ coupled with (4.3) provides an elementary proof of the strong continuity of $\underset{\sim p}{E_{p}}(\lambda)$ on $\mathbf{R}$. It is not at all clear that this result, although naturally expected, could have been 
addressed within the context of translation groups' theory (as its counterpart in $\left.H^{p}(\mathrm{D})\right)$. The hyperbolic case, on the other hand, we have not been able to address in a similar straightforward way (i.e. without appealing to Lemma (3.2) and Lemma (4.6)).

We conclude this paper with a description of the action of $E_{p}(\lambda), \lambda \in \mathbf{R}$, on the various components of $H^{p}\left(\mathbf{T}^{2}\right)$ (described in Lemma (4.5)), as a corollary to Theorem (4.9)(ii). Special emphasis is given on $E_{2}(\lambda), \lambda \in \mathbf{R}$, for which although in the context of Hilbert spaces, very little was known about its behavior.

Corollary (4.10). The following are true:

(i) $\left.\underset{\sim p}{E_{p}}(\lambda)\right|_{Z_{p} \oplus \wedge_{1}^{p}}=I_{Z_{p} \oplus \wedge_{1}^{p}}$ for $\lambda \geq 0,1<p<+\infty$;

$$
\begin{aligned}
& \underset{\sim p}{E_{p}}(\lambda)\left(z_{1}^{-m} z_{2}^{n}\right) \\
& \quad=z_{1}^{-m} z_{2}^{n}\left\{1-e^{-\lambda} f_{m}\left(z_{1}\right) \xi_{\lambda}\left(z_{1}\right) \sum_{\substack{0 \leq \alpha+\beta \leq m-1 \\
\alpha \geq 0, \beta \geq 0}}\left[\begin{array}{c}
m-2 / p \\
\beta
\end{array}\right] \frac{(-2 \lambda)^{\alpha}}{\alpha !}\left[\frac{z_{1}}{1-z_{1}}\right]^{\alpha+\beta}\right\}
\end{aligned}
$$

for $m \geq 1, n \geq 1, \lambda \geq 0$ and a.a. $\left(z_{1}, z_{2}\right) \in \mathbf{T}^{2}$;

(iii) $\left.\underset{\sim}{E_{2}}(\lambda)\right|_{\wedge_{2}^{2}}=0$ for $\lambda<0$;

(iv) $\underset{\sim}{E_{2}}(\lambda)\left(z_{1}^{m} z_{2}^{n}\right)=z_{2}^{n} e^{\lambda} \xi_{\lambda}\left(z_{1}\right) \sum_{\substack{0 \leq \gamma+\delta \leq m-1 \\ \gamma \geq 0, \delta \geq 0}}(-1)^{\gamma}\left[\begin{array}{c}m \\ \gamma\end{array}\right] \frac{(-2 \lambda)^{\delta}}{\delta !}\left(1-z_{1}\right)^{m-(\gamma+\delta)}$ for $m \geq 0, n \geq 0, \lambda<0$ and a.a. $\left(z_{1}, z_{2}\right) \in \mathbf{T}^{2}$.

Proof. (Notice that the definitions of $f_{m}(z)$ and $\xi_{\lambda}(z)$ given in $\S 3$ are still meaningful on $\mathbf{T} \backslash\{1\}$ and, as such, were employed in this corollary.)

(i) The result $V_{\lambda}=I, \lambda \geq 0$, of Theorem (3.5)(i) and the "identification" between $Z^{p}$ and $H^{p}(\mathbf{T})$, coupled with the mean convergence theorem (see e.g.

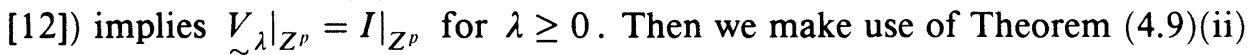
and we are done with the $Z^{p}$ component. We thus need only to demonstrate now $\left.\underset{\sim}{V}\right|_{\Lambda_{1}^{p}}=\left.I\right|_{\Lambda_{1}^{p}}$ for $\lambda \geq 0$; it will suffice evidently to show $\underset{\sim}{V}\left(z_{1}^{m} z_{2}^{n}\right)=z_{1}^{m} z_{2}^{n}$ for $m \geq 0, n \geq 1$ and $\lambda \geq 0$.

We will employ the same contour, $\mathscr{C}$ in the UHP as in the proof of Theorem (3.5)(i). We can easily see that

$$
\underset{\sim}{V}\left(z_{1}^{m} z_{2}^{n}\right)=z_{2}^{n} \underset{\sim \lambda}{V_{\lambda}} z_{1}^{m}=z_{2}^{n} \lim _{\varepsilon \rightarrow 0+} \frac{1}{i \pi} \int_{\varepsilon<|t| 1 / \varepsilon} \frac{e^{i \lambda t}}{t} \frac{\left(z_{1}-\frac{i}{2}\left(1-z_{1}\right) t\right)^{m}}{\left(1-\frac{i}{2}\left(1-z_{1}\right) t\right)^{m+2 / p}} d t
$$

for a.a. $\left(z_{1}, z_{2}\right) \in \mathbf{T}^{2}$ (in particular consider $z_{1} \neq 1$ ). Observe also that the equation $1-\frac{i}{2}\left(1-z_{1}\right) w=0$, for given $z_{1} \in \mathbf{T} \backslash\{1\}$, solved in $\mathbf{C}$ has the root $w=w_{1}=2 / i\left(1-z_{1}\right)$ and $\operatorname{Im} w_{1}=-1$, so that $w_{1} \notin$ UHP. In addition it is easy to check that $1-\frac{i}{2}\left(1-z_{1}\right) w \notin\{\zeta: \operatorname{Re} \zeta \leq 0$ and $\operatorname{Im} \zeta=0\}$. In 
conclusion the expression $\left\{1-\frac{i}{2}\left(1-z_{1}\right) w\right\}^{m+2 / p}$ is well defined as its usual analytic branch for $w$ in the UHP and its boundary. Using now

$$
\int_{\mathscr{C}} \frac{e^{i \lambda w}}{w} \frac{\left(z_{1}-\frac{1}{2}\left(1-z_{1}\right) w\right)^{m}}{\left(1+\frac{i}{2}\left(1-z_{1}\right) w\right)^{m+2 / p}} d w=0
$$

and proceeding as in Theorem (3.5)(i), we see that the integral in (4.11) can be evaluated as follows:

$$
\begin{aligned}
& \lim _{\varepsilon \rightarrow 0+} \frac{1}{i \pi} \int_{l_{1} \cup l_{2}} \frac{e^{i \lambda t}}{t} \frac{\left(z_{1}-\frac{i}{2}\left(1-z_{1}\right) w\right)^{m}}{\left(1-\frac{i}{2}\left(1-z_{1}\right) t\right)^{m+2 / p}} d t \\
& \quad=\frac{1}{i \pi}\left\{i \pi \lim _{|w|=\varepsilon \rightarrow 0+} \frac{e^{i \lambda w}\left(z_{1}-\frac{i}{2}\left(1-z_{1}\right) w\right)^{m}}{\left.\left(1-\frac{i}{2}\left(1-z_{1}\right)\right) w\right)^{m+2 / p}}\right\}=z_{1}^{m}
\end{aligned}
$$

for $m \geq 0$. Q.E.D.

(ii) Working as in (i) we see that $\left(1-\frac{i}{2}\left(1-z_{1}\right) w\right)^{m 2 / p}$ has a well-defined analytic branch in the UHP and its boundary, the equation $z_{1}-\frac{i}{2}\left(1-z_{1}\right) w=0$ has a root $w_{2}=2 z_{1} / i\left(1-z_{1}\right) \in$ UHP since $\operatorname{Im} w_{2}=1$, and that

$$
V_{\sim} z_{1}^{-m} z_{2}^{n} \lim _{\varepsilon \rightarrow 0+} \frac{1}{i \pi} \int_{l_{1} \cup l_{2}} \frac{e^{i \lambda t}}{t} \frac{\left(1-\frac{i}{2}\left(1-z_{1}\right) w\right)^{m-2 / p}}{\left(z_{1}-\frac{i}{2}\left(1-z_{1}\right) w\right)^{m}} d w .
$$

Evidently $w_{2}$ is a pole of the integrand of (4.12) of multiplicity $m$. We thus have

$$
\underset{\sim}{V}\left(z_{1}^{-m} z_{2}^{n}\right)=z_{2}^{n} \frac{1}{i \pi}\left\{i \pi \lim _{|w|=\varepsilon \rightarrow 0+} e^{i \lambda w} \frac{\left(1-\frac{i}{2}\left(1-z_{1}\right) w\right)^{m-2 / p}}{\left(z_{1}-\frac{i}{2}\left(1-z_{1}\right) w\right)^{m}}+0+2 \pi i \operatorname{Res}\left(z_{1}\right)\right\}
$$

which implies $V_{\lambda}\left(z_{1}^{-m} z_{2}^{n}\right)=z_{1}^{-m} z_{2}^{n}+2 z_{2}^{n} \operatorname{Res}\left(z_{1}\right)$ for a.a. $\left(z_{1}, z_{2}\right) \in \mathbf{T}^{2}$, where by $\operatorname{Res}\left(z_{1}\right)$ we denote the residue of

$$
\frac{e^{i \lambda w}}{w} \frac{\left(1-\frac{i}{2}\left(1-z_{1}\right) w\right)^{m-2 / p}}{\left(z_{1}-\frac{i}{2}\left(1-z_{1}\right) w\right)^{m}}
$$

at $w=w_{2}$. By definition now and after elementary manipulations (see [16, pp. 55-56] for details) we get

$$
\operatorname{Res}\left(z_{1}\right)=\left[\frac{2 i}{1-z_{1}}\right]^{m} a_{m-1}(z),
$$

where $a_{m-1}(z)$ is the $(m-1)$ th Taylor coefficient of

$$
f(w)=\left(\frac{e^{i \lambda w}}{w}\right)\left(1-\frac{i}{2}\left(1-z_{1}\right) w\right)^{m-2 / p}
$$

expanded near $w=w_{2}$. Observe next that

$$
1-\frac{i}{2}\left(1-z_{1}\right) w=\left(1-z_{1}\right)\left(1-\frac{i}{2}\left(w-w_{2}\right)\right)
$$


and that each factor has a positive real part when $\operatorname{Im} w \geq 0, z_{1} \in \mathbf{T} \backslash\{1\}$. Thus

$$
f(w)=e^{2 \lambda z_{1} /\left(1-z_{1}\right)} \frac{e^{i \lambda\left(w-w_{2}\right)}}{w}\left(1-\frac{i}{2}\left(w-w_{2}\right)\right)^{m-2 / p} .
$$

Expanding the functions of $w$ involved in (4.15) near $w_{2}$ and after collecting the coefficients of $\left(w-w_{2}\right)^{k}$ for $k=0,1,2, \ldots$ we conclude that

$$
\begin{aligned}
& a_{m-1}\left(z_{1}\right) \\
& \quad=\frac{i\left(1-z_{1}\right)}{2 z_{1}} e^{2 \lambda z_{1} / 2 z_{1}}\left(1-z_{1}\right)^{m-2 / p} \sum_{\substack{\alpha+\beta+\gamma=m-1 \\
\alpha \geq 0, \beta \geq 0, \gamma \geq 0}} \frac{(i \lambda)^{\alpha}}{\alpha !}\left[\begin{array}{c}
m-2 / p \\
\beta
\end{array}\right] \frac{(-1)^{\gamma}}{\left(z_{i}\right)^{\beta} w_{2}^{\gamma}}
\end{aligned}
$$

Combining (4.13), (4.14) and (4.16) we obtain the stated result.

(iii) For $p=2$ and $\lambda<0$ we simply employ the contour $\mathscr{C}$ (travelled c.w.) in the LHP, as it was used in the proof of Theorem (3.5)(iii). By definition of $\Lambda_{2}^{2}$ it will suffice to show $\underset{\sim}{E_{2}}(\lambda)\left(z_{1}^{-m} z_{2}^{n}\right)=0$ for $m \geq 1, n \geq 1$ and a.a. $\left(z_{1}, z_{2}\right) \in \mathbf{T}^{2}$ or, using Theorem (4.9)(ii), $V_{\lambda}\left(z_{1}^{-m} z_{2}^{n}\right)=-z_{1}^{m} z_{2}^{n}$ for $m \geq$ $1, n \geq 1$ and a.a. $\left(z_{1}, z_{2}\right) \in \mathbf{T}^{2}$. Working as before we have that

$$
V_{\sim}\left(z_{1}^{-m} z_{2}^{n}\right)=z_{2}^{n} \lim _{\varepsilon \rightarrow 0+} \frac{1}{i \pi} \int_{l_{1} \cup l_{2}} \frac{e^{i \lambda t}}{t} \frac{\left(1-\frac{i}{2}\left(1-z_{1}\right) t\right)^{m-1}}{\left(z_{1}-\frac{i}{2}\left(1-z_{1}\right) t\right)^{m}} d t
$$

and the integrand in (4.17) can be calculated directly now since the only pole in $\{w \in \mathbf{C}: \operatorname{Im} w \leq 0\}$ is at $w=0$. Thus (4.17) implies

$$
i \pi V_{\lambda}\left(z_{1}^{-m} z_{2}^{n}\right)+z_{2}^{n} i \pi \lim _{\varepsilon=|w| \rightarrow 0+} e^{i \lambda w} \frac{\left(1-\frac{i}{2}\left(1-z_{1}\right) w\right)^{m-1}}{\left(z_{1}-\frac{i}{2}\left(1-z_{1}\right) w\right)^{m}}+0=0
$$

which leads to ${\underset{\sim}{\lambda}}_{\lambda}\left(z_{1}^{-m} z_{2}^{n}\right)=-z_{1}^{-m} z_{2}^{n} \quad$ Q.E.D.

(iv) $\underset{\sim}{E_{2}}(\lambda)\left(z_{1}^{m} z_{2}^{n}\right)=z_{2}^{n} E_{\sim}(\lambda) z_{1}^{m}=z_{2}^{n}{\underset{\sim}{2}}_{2}(\lambda)\left[\sum_{k=0}^{m}(-1)^{k}\left[\begin{array}{c}m \\ k\end{array}\right] f_{k+1}\left(z_{1}\right)\right]=$ $z_{2}^{n} \sum_{k=0}^{m}(-1)^{k}\left[\begin{array}{c}m \\ k\end{array}\right]{\underset{\sim}{2}}_{2}(\lambda) f_{k+1}\left(z_{1}\right)$ and we appeal now to Theorem (3.5)(iii) with $p=2$ and allowing $z \rightarrow z_{1}$ for $z \in \mathbf{D}$ and $z_{1} \in \mathbf{T} \backslash\{1\}$.

Remarks. (1) From (4.13) we can see now that $\underset{\sim}{E_{p}}(\lambda) \neq I$ for any $\lambda \geq 0$, behavior drastically different from the behavior of its counterpart on $H^{p}(\mathbf{D})$.

(2) We could arrive at (iv) of Corollary (4.10) using contour integration as in (i).

\section{REFERENCES}

1. H. Benzinger, E. Berkson, and T. A. Gillespie, Spectral families of projections, semigroups and differential operators, Trans. Amer. Math. Soc. 275 (1983), 431-475.

2. E. Berkson, Spectral families of projections in Hardy spaces, J. Funct. Anal. 60 (1985), 146-167. 
3. E. Berkson and T. A. Gillespie, The generalized M. Riesz theorem and transference, Pacific J. Math. 120 (1985), 279-288.

4. __, Steckkin's theorem, transference, and spectral decompositions, J. Funct. Anal. 70 (1987), 140-170.

5. E. Berkson. T. A. Gillespie, and P. S. Muhly, Abstract spectral decompositions guaranteed by the Hilbert transform, Proc. London Math. Soc. (3) 53 (1986), 489-517.

6. E. Berkson, R. Kaufman, and H. Porta, Möbius transformations of the disk and one-parameter groups of isometries of $H^{p}$, Trans. Amer. Math. Soc. 199 (1974), 223-239.

7. E. Berkson and $\mathrm{H}$. Porta, Hermitian operators and one-parameter groups of isometries in Hardy space, Trans. Amer. Math. Soc. 185 (1973), 331-344.

8. _ One parameter groups of isometries on Hardy spaces of the torus, Trans. Amer. Math. Soc. 220 (1976), 373-391.

9. D. L. Burkholder, A geometric condition that implies the existence of certain singular integrals of Banach-spac-evalued functions, Proc. Conf. Harmonic Analysis in Honor of Antoni Zygmund (Chigago, Ill., 1981) (W. Beckner et al., eds.), Wadsworth, Belmont, Calif., 1983.

10. N. Dunford and J. T. Schwartz, Linear operators. I. General theory, Pure and Appl. Math., Vol. 7, Interscience, New York, 1958.

11. H. R. Dowson, Spectral theory of linear operators, London Math. Soc. Monographs, No. 12, Academic Press, New York, 1978.

12. P. L. Duren, Theory of $H^{p}$ spaces, Pure and Appl. Math., Vol. 38, Academic Press, New York, 1970 .

13. F. Forelli, The isometries of $H^{p}$, Canad. J. Math. 16 (1964), 721-728.

14. J. B. Garnett, Bounded analytic functions, Pure and Appl. Math., Vol. 96, Academic Press, New York, 1981.

15. K. Hoffman, Banach spaces of analytic functions, Prentice-Hall, Englewood Cliffs, N.J., 1962.

16. D. Karayannakis, Spectral decompositions of isometries on Hardy spaces of the disk and the torus, Doctoral Dissertation, Univ. of Illinois at Urbana-Champaign, 1986.

17. Y. Katznelson, An introduction to harmonic analysis, Dover, New York, 1976.

18. P. Koosis, Introduction to $H_{p}$ spaces, London Math. Soc. Lecture Notes Series, no. 40, Cambridge Univ. Press, London and New York, 1980.

19. N. Lal and S. Merrill, Characterization of certain invariant subspaces of $H^{p}$ and $L^{p}$ spaces derived from logmodular algebras, Pacific J. Math. 30 (1969), 463-474.

20. F. Riesz and B. Sz-Nagy, Functional analysis, Ungar, New York, 1955.

21. Y. R. Ringrose, On well-bounded operators II, Proc. London Math. Soc. (3) 13 (1963), 613638.

22. D. Sarason, A remark on the Volterra operator, J. Math. Anal. Appl. 12 (1965), 244-246.

Department of Mathematics, University of Illinois, Urbana, Illinois 61801

Current address: Department of Mathematics, University of Crete, P.O. Box 470, Iraklion, Crete, Greece 